\title{
A narrative review of the emerging role of lymphocyte antigen 6 complex locus $K$ in cancer: from basic research to clinical practice
}

\author{
Dandan Guo ${ }^{1}$, Yujia Liu ${ }^{1}$, Yuchen Jiang', Shuilian Zheng ${ }^{1}$, Tong Xu' ${ }^{1}$, Jiazhen Zhu ${ }^{2}$, Pengcheng Chen ${ }^{3}$, \\ Ping Huang ${ }^{1,4}$, Yiwen Zhang ${ }^{1,4}$ \\ ${ }^{1}$ Clinical Pharmacy Center, Department of Pharmacy, Zhejiang Provincial People's Hospital, Affiliated People's Hospital of Hangzhou Medical \\ College, Hangzhou, China; ${ }^{2}$ School of Pharmaceutical Sciences, Zhejiang Chinese Medical University, Hangzhou, China; ${ }^{3}$ Department of Thoracic \\ Surgery, Zhejiang Provincial People's Hospital, Affiliated People’s Hospital of Hangzhou Medical College, Hangzhou, China; ${ }^{4}$ Key Laboratory of \\ Endocrine Gland Diseases of Zhejiang Province, Hangzhou, China \\ Contributions: (I) Conception and design: Y Zhang, P Huang; (II)Administrative support: None; (III) Provision of study materials or patients: None \\ (IV) Collection and assembly of data: S Zheng, T Xu, J Zhu; (V) Data analysis and interpretation: D Guo, Y Liu, Y Jiang; (VI) Manuscript writing: \\ All authors; (VII) Final approval of manuscript: All authors. \\ Correspondence to: Yiwen Zhang. Clinical Pharmacy Center, Department of Pharmacy, Zhejiang Provincial People's Hospital, Affiliated People's \\ Hospital of Hangzhou Medical College, 158 Shangtang Road, Gongshu District, Hangzhou 310014, China. Email: zjzyw2003@163.com.
}

\begin{abstract}
Objective: In this paper, we will discuss the structure, function and role of lymphocyte antigen 6 complex locus $\mathrm{K}(\mathrm{LY} 6 \mathrm{~K})$ in disease model, and highlight the new progress of LY6K in current clinical trials. It provides reference value for future basic research.

Background: Cancer is a global public health problem that must be solved. The ability of tumor cells to evade the antitumor immune response makes cancer research and treatment more difficult. LY6K is highly expressed in a variety of cancers, can stimulate the immune system, and has tumor specificity. At present, a large number of vaccines have entered clinical trials.

Methods: The PubMed, umin.ac.jp/ctr and Clinical Trials.gov databases were searched for LY6K published literature and clinical trials. The structure and function of LY6K and the current state of research on LY6K in human cancers were discussed to provide reference for further research.

Conclusions: The $L Y 6 K$ gene has been shown to be highly expressed in a variety of tumor cells, driving tumorigenesis and progression, and is negatively correlated with poor prognosis in patients. At the same time, LY6K can stimulate the immune response of the body's immune cells. The study of LY6K-related vaccines in clinical trials also suggests that targeting LY6K may be a new direction for tumor immunotherapy.
\end{abstract}

Keywords: Lymphocyte antigen 6 complex locus K (LY6K); cancer; immunotherapy; vaccines

Submitted Oct 19, 2021. Accepted for publication Dec 02, 2021.

doi: 10.21037/atm-21-5831

View this article at: https://dx.doi.org/10.21037/atm-21-5831

\section{Introduction}

The $L y 6$ gene family is at the interface of stem cell biology and cancer biology. The Ly6 complex was first described in $1977(1,2)$. More than 20 structurally related genes have been identified in the Ly6 complex on chromosome 15 (3). Ly6 proteins, most of which are membrane-bound molecules attached to the cell surface through c-terminal glycosylphosphatidylinositol (GPI) anchoring, share a conserved motif called the LU domain (3). It has been reported that members of the Ly6/uPAR superfamily, LY6D, LY6E, LY6H, LY6K, PSCA, LYPD2, SLURP1, GML, GPIHBP1 and LYNX1, play roles in cell proliferation, migration, cell-cell interaction, immune regulation, macrophage activation, and cytokine secretion. Some 
members of the Ly6/uPAR superfamily are abnormally expressed in cancer and negatively correlated with patient prognosis (4). Furthermore, the expression of members of Ly6/uPAR superfamily in immune cells has been confirmed, indicating that Ly6/uPAR superfamily plays an important role in tumor immune regulation and may be a potential target for tumor diagnosis and treatment $(4,5)$.

A novel member of the Ly6/uPAR superfamily-LY6K, also known as cancer-testis antigen (6), is related to the murine stem cell antigen-1 gene. LY6K is overexpressed in a variety of human malignancies, including lung cancer, breast cancer, colorectal cancer, bladder cancer, and esophageal carcinoma (7-12), whereas its expression is low or difficult to detect in corresponding normal tissues. This overexpression of LY6K is closely related to invasive growth and enhanced mobility in several tumor types, as well as poor prognosis and recurrence $(10,12)$. Several studies have confirmed that high LY6K expression is associated with poor prognosis in esophageal squamous cell carcinoma, non-small cell lung cancer, and breast cancer $(8,12,13)$. This suggests that LY6K may play a role in tumor recurrence and metastasis (12). In addition, the role of LY6K in tumor immunity is also widely favored. For more than a century, researchers have been studying how to use the immune response to eliminate tumors (14). For example, depletion of dendritic cells (DC) can inhibit tumor growth $(15,16)$; meanwhile, a part of immune cells can also recognize viral infection and tumor cell targets by activating or inhibiting cell surface receptors; and NK cells can also secrete a large number of cytokines that directly or indirectly kill tumor cells $(17,18)$. A recent study showed that LY6K can induce an immune response that is involved in human malignancies. Overexpression of LY6K can inhibit the maturity and development of T cells (19). In other words, knocking out LY6K can induce $\mathrm{T}$ cell activation and stimulate cellular immunity in the body. Additionally, its induced immune response is tumor specific. According to the above analysis, LY6K is a potential therapeutic target for cancer immunotherapy $(9,12,20-22)$.

In this review, we will focus on the role of LY6K. We know that LY6K is overexpressed in tumors and is associated with poor prognosis of patients. However, the molecular mechanism of its action remains unclear. Its relationship with tumor immune escape has not been clearly elucidated. Therefore, we will discuss the latest research progress, current research status and future development possibilities of LY6K. It will lay a theoretical foundation for future research and provide new research ideas. We are committed to exploring new targets for the treatment of cancer and providing new choices for the clinical treatment of cancer patients. We present the following article in accordance with the Narrative Review reporting checklist (available at https://dx.doi.org/10.21037/atm-21-5831).

\section{LY6K regulation and function}

\section{Structure and function}

LY6K was initially identified as an unannotated transcript $(12,23)$ and eventually identified as a member of the Ly6/ uPAR protein superfamily by bioinformatics (24). LY6K is located on chromosome 8q24.3, and is about $1735 \mathrm{bp}$ in size. Its transcript encompasses an open reading frame of 5,261 bases that encodes 4 homologous proteins (23,25-27). One of these proteins is a 165 -amino acid transmembrane protein. It consists of 3 exons, of which the third is the largest, and, like other Ly6 antigens, there are a total of 10 cysteine residues in a shared motif (6). LY6K contains sequences that encode GPI anchoring that should result in its localization to the cell membrane, although whether this occurs is unknown $(9,28)$.

\section{Regulation of LY6K expression}

LY6K is a cancer/testicular antigen that is highly expressed in cancer cells and tissues but is not expressed in normal tissues other than the testis. Several groups have shown that the expression of LY6K is increased in cancer, including cervical cancer and head and neck squamous cell cancer $(29,30)$.

Some studies have demonstrated that the JunD and Fra1 transcription factors bind to the LY6K promoter to regulate its transcription in vitro (31). The regulatory mechanism of LY6K is shown in Figure 1. Activated JunD and Fra1 promote LY6K expression at the mRNA and protein levels. Furthermore, the methylation of the SNP242c allele and a CpG site near the activating protein-1 (AP-1) binding site reduce the binding of AP-1 to the promoter by attracting PAX3 and interfering with AP-1 binding, leading to the downregulation of LY6K (31). Meanwhile, the interaction between LY6K and AP-1 enhances extracellular signalregulated kinase (ERK) signaling, which ultimately promotes the occurrence of tumors. The occurrence of tumors can enhance the expression of the AP- 1 transcription factor, further affecting the expression regulation of the Jun family, ultimately impacting the biological function. 


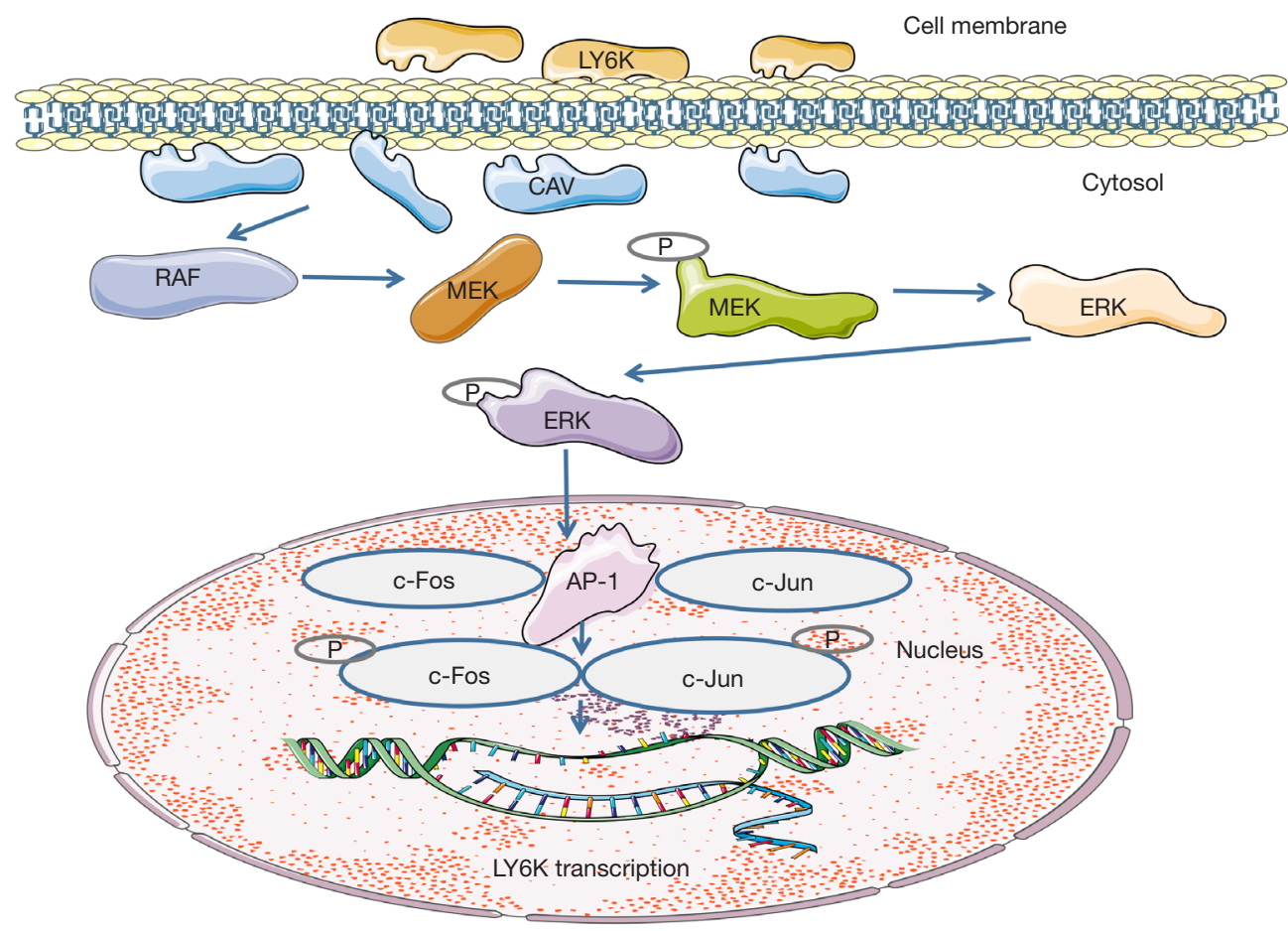

Figure 1 The downstream regulatory mechanisms of lymphocyte antigen 6 complex locus K (LY6K). LY6K regulates the occurrence and development of cancers by regulating Caveolin 1 (CAV-1), extracellular signal-regulated kinase (ERK), and activating protein-1 (AP-1).

\section{The role of LY6K in cancers}

Little is known about the function of LY6K in the development of cancer. Most current studies on LY6K in human diseases have explored its prognostic ability in cancer. As shown in Figure 2, the expression of LY6K is closely related to overall survival in various cancers (data from http://kmplot.com). LY6K is regulated downstream of ERK signaling to increase the tumorigenicity of glioblastoma in vitro and in vivo (32). In addition, LY6K has been reported to be upregulated in metastatic ER-positive breast cancer $(31,33,34)$, lung cancer $(12,13)$, esophageal squamous cell carcinoma (35), gingival and buccal carcinoma (30), and bladder cancer (11) in preclinical and clinical studies.

Some studies have used the double antibody sandwich enzyme-linked immunosorbent assay (DAS-ELISA) method to detect the expression of LY6K in the serum of patients with early non-small cell lung cancer. LY6K is significantly upregulated in these patients compared to healthy individuals. Therefore, LY6K may be an important biomarker for the early diagnosis of non-small cell lung cancer (24). Another study found a significant difference in the expression of LY6K mRNA between bladder cancer $(\mathrm{n}=91)$ and normal control $(\mathrm{n}=37)$ samples $(11,23)$.

Kono et al. suggest that LY6K can induce T cells to produce specific immune responses (36). Patient killer $\mathrm{T}$ cells have been shown to kill cancer cells with specific antigens in vitro (36). Knockout of LY6K in overexpressed tumor cell lines could inhibit the directed migration of a variety of tumor cell lines, and this phenomenon shows that LY6K plays an important role in tumor immunotherapy and may be a potential therapeutic target for small-molecule inhibitors (36). The role of LY6K in tumor immune escape is related to NK cell binding, cytokine release, and the programmed cell death ligand 1/programmed death-1 (PDL1/PD-1) checkpoint. Its potential mechanism involves constitutive TGF signal transduction and the interaction between tumor cells and the tumor microenvironment, especially tumor immune surveillance (8).

Based on the above recent studies, it can be concluded that LY6K is a potential tumor-related marker. Meanwhile, it also suggests that LY6K is a potential target for cancer immunotherapy. 


Tumor typc
Pheochromocytoma and paraganglioma
Esophageal squamous cell carcinoma
Pancreatic ductal adenocarcinoma
Bladder carcinoma
Liver hepatocellular carcinoma
Breast cancer
Ovarian cancer
Head-neck squamous cell carcinoma
Stomach adenocarcinoma
Lung squamous cell carcinoma
Sarcoma
Kidney renal clear cell carcinoma
Lung adenocarcinoma
Cervical squamous cell carcinoma
Thymoma
Rectum adenocarcinoma
Thyroid carcinoma
Kidney renal papillary cell carcinoma
Uterine corpus endometrial carcinoma
Esophageal adcnocarcinoma

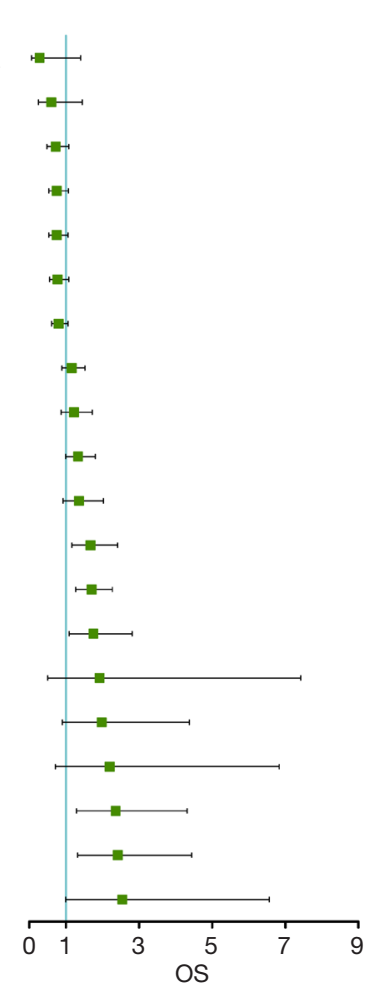

$\begin{array}{cc}\text { HR }(95 \% \mathrm{CD}) \text {-(high vs low) } & \text { logrank P } \\ 0.28(0.06-1.4) & 0.0982 \\ 0.6(0.25-1.45) & 0.2533 \\ 0.72(0.48-1.08) & 0.1082 \\ 0.75(0.53-1.07) & 0.1072 \\ 0.75(0.53-1.06) & 0.098 \\ 0.77(0.55-1.08) & 0.1292 \\ 0.8(0.61-1.06) & 0.1261 \\ 1.16(0.89-1.52) & 0.2717 \\ 1.22(0.87-1.72) & 0.2531 \\ 1.33(0.99-1.8) & 0.0595 \\ 1.36(0.92-2.02) & 0.1245 \\ 1.67(1.16-2.41) & 0.0051 \\ 1.7(1.27-2.27) & 0.0003 \\ 1.75(1.09-2.81) & 0.0194 \\ 1.92(0.5-7.42) & 0.3374 \\ 1.98(0.9-4.38) & 0.0852 \\ 2.2(0.71-6.83) & 0.1607 \\ 2.36(1.29-4.31) & 0.0041 \\ 2.42(1.32-4.44) & 0.0032 \\ 2.54(0.99-6.56) & 0.0451\end{array}$

Figure 2 The overall survival risk ratio of lymphocyte antigen 6 complex locus K (LY6K) in a pan-cancer series.

\section{Clinical development of therapeutic agents to target LY6K in cancer}

Current treatment measures mainly include surgical treatment, chemotherapy, radiotherapy, traditional Chinese medicine, cryoablation, and immunotherapy for cancer. At present, there are several experimental drugs for LY6K in clinical trials for a variety of human cancers and other diseases (Table 1; https://clinicaltrials.gov/ and https://www. umin.ac.jp/ctr). This section will focus on LY6K-related drugs developed to take advantage of high LY6K expression in tumors.

The main types of cancer vaccines include protein or synthetic peptides of cancer antigens, tumor antigens, DNA/ RNA encoding cancer antigens, and other types (55). Cancer vaccines have been widely used in preclinical and clinical research in various malignant tumors, and have also made some achievements. For example, Mycobacterium bovis bacillus Calmette-Guérin (BCG) vaccination improved the activation and failure of tumor specific $\mathrm{T}$ cells, so as to achieve the purpose of treating certain types of bladder cancer (56). In a phase I clinical study of melanoma, the results of a clinical trial of an RNA cancer vaccine showed metastatic regression or stable disease in patients, indicating the vaccine's inhibitory effect on the tumor (57). Gp100 peptide vaccine has also been studied in combination with high doses of the cytokine interleukin-2 (IL-2) in melanoma patients. Results from this trial showed significant improvements in objective response rates and progressionfree survival $(\mathrm{PFS})$ in patients treated with the combination compared to patients treated with IL-2 alone (58).

In patients with advanced esophageal squamous cell carcinoma, the pDC and NK cell populations increased and the antigen-specific CD $8+T$ cell response was successfully activated after administration of the epitope peptide (level 1) combined with the CPG-7909 (level $2 / 3$ ) vaccine (22). In addition, interferon-alpha (IFN- $\alpha$ ) and related chemokines were upregulated, and the corresponding NK cells were activated (22). Therefore, the vaccine enhanced not only tumor-specific acquired immunity but also innate immunity (22).

With regard to HLA-A24 and HLA-A24-related vaccines, in a phase I trial, an immune response was observed in 13 of 15 lung cancer patients, and some patients had stable disease for at least 2 months (7). In a phase I 
Table 1 Clinical trials of lymphocyte antigen 6 complex locus K (LY6K)-related interventions

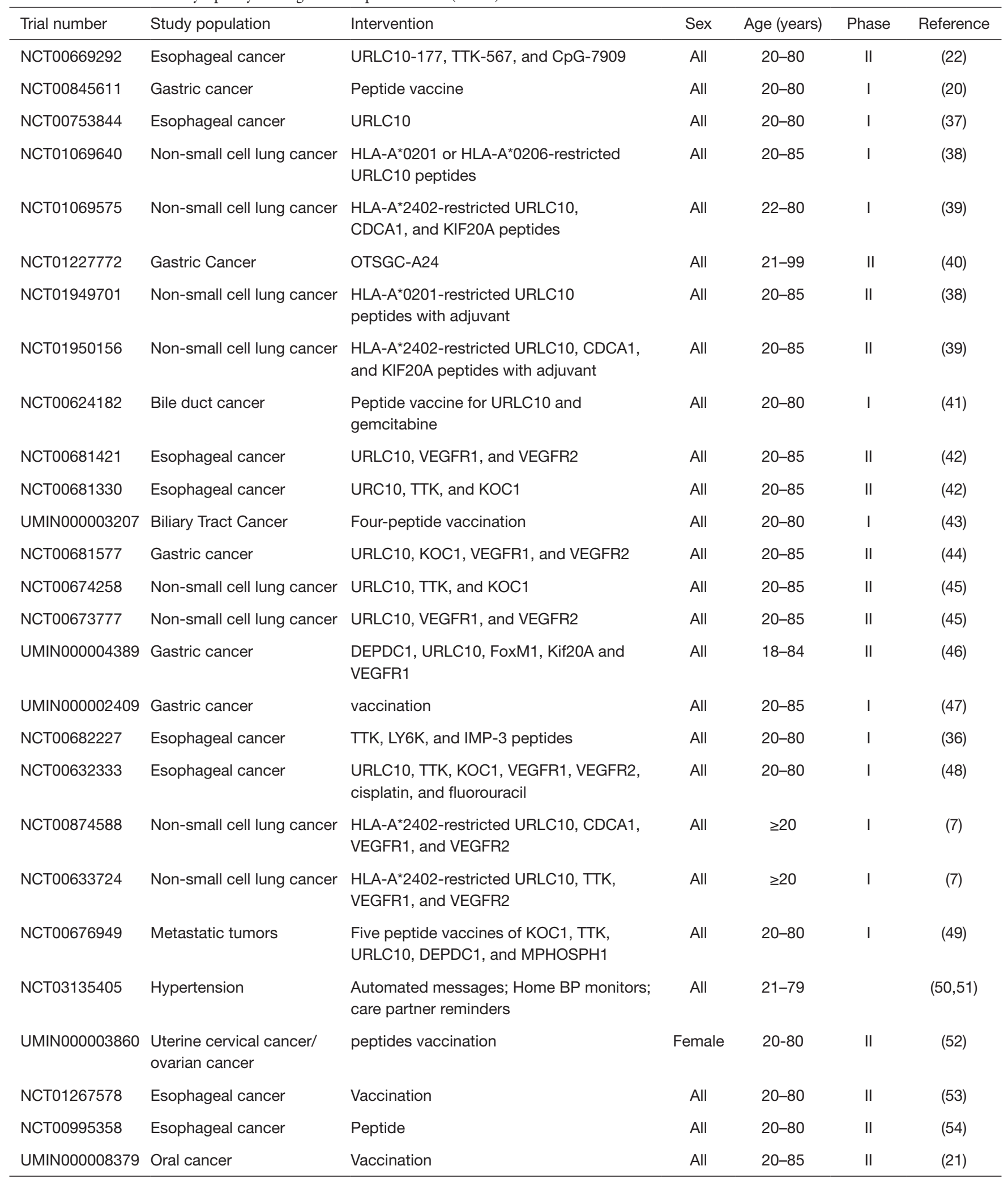


clinical trial in which 6 patients with advanced gastric cancer were administered an HLA-A24-related LY6K-177 peptide vaccine, 3 patients had stable disease and 1 patient had a partial response, and the average total survival time reached 7.9 months. This greatly improved the overall survival rate of gastric cancer patients. Furthermore, the vaccine had few toxic side effects and was well tolerated (20). A clinical phase II trial of the LY6K-177 polypeptide vaccine for head and neck squamous cell carcinoma showed that the vaccine induced an immune response and improved prognosis in patients with advanced HNSCC and was well tolerated (21).

According to the above findings, with the increasing variety of diseases, people do not have safer and more effective treatment means to actively treat. The advent of vaccines has greatly reduced the fear of cancer and other diseases. At present, tumor vaccines have received more and more attention, and a large number of vaccines are in the clinical phase II or phase III trials stage, which can prolong the PFS time and improve the quality of life of patients.

\section{Conclusions}

LY6K is a potential target for the treatment of various cancers, and explorations of its biological function have only just begun. The best method for optimizing LY6K in tumor therapy and tumor immunotherapy is still an urgent problem to be solved. It is necessary to better understand the working mechanism of LY6K as a secreted membrane protein to enhance the progress of tumor-related vaccine clinical work, which may provide important information for the future development of cancer treatment. We must also clarify the interaction between LY6K and immune cells and characterize the interaction between LY6K and immune checkpoints (PD-L1). This will provide a solid foundation for further research.

\section{Acknowledgments}

Funding: This study was supported by Zhejiang Provincial Natural Science Foundation (grant number: LY20H310001 and LQ19H280004), the Medical and Health Research Program of Zhejiang Province (grant number: 2021KY040, 2021KY491, 2022KY069 and 2022RC008), Chinese Medicine Research Program of Zhejiang Province (grant number: 2021ZZ001), the Zhejiang Provincial Program for the Cultivation of New Heath Talents (to YZ) and "10000 Talents Plan" of Zhejiang Province (to $\mathrm{PH}$ ).

\section{Footnote}

Reporting Checklist: The authors have completed the Narrative Review reporting checklist. Available at https:// dx.doi.org/10.21037/atm-21-5831

Conflicts of Interest: All authors have completed the ICMJE uniform disclosure form (available at https:// dx.doi.org/10.21037/atm-21-5831). YL reports personal fees from the Medical and Health Research Program of Zhejiang Province (2022RC008). Tong Xu reports personal fees from the Medical and Health Research Program of Zhejiang Province (2021KY491). JZ reports personal fees from the Zhejiang Provincial Natural Science Foundation (LQ19H280004). Ping Huang reports personal fees from the "10000 Talents Plan" of Zhejiang Province. YZ reports personal fees from the Zhejiang Provincial Program for the Cultivation of New Heath Talents, personal fees from the Zhejiang Provincial Natural Science Foundation (LY20H310001), personal fees from the Chinese Medicine Research Program of Zhejiang Province (2021ZZ001), and the Medical and Health Research Program of Zhejiang Province (2022KY069 and 2021KY040). The other authors have no conflicts of interest to declare.

Ethical Statement: The authors are accountable for all aspects of the work in ensuring that questions related to the accuracy or integrity of any part of the work are appropriately investigated and resolved.

Open Access Statement: This is an Open Access article distributed in accordance with the Creative Commons Attribution-NonCommercial-NoDerivs 4.0 International License (CC BY-NC-ND 4.0), which permits the noncommercial replication and distribution of the article with the strict proviso that no changes or edits are made and the original work is properly cited (including links to both the formal publication through the relevant DOI and the license). See: https://creativecommons.org/licenses/by-nc-nd/4.0/.

\section{References}

1. McKenzie IF, Gardiner J, Cherry M, et al. Lymphocyte antigens: Ly-4, Ly-6, and Ly-7. Transplant Proc 1977;9:667-9.

2. Woody JN, Feldmann M, Beverley PC, et al. Expression of alloantigens LY-5 and LY-6 on cytotoxic effector cells. J Immunol 1977;118:1739-43. 
3. Rock KL, Reiser H, Bamezai A, et al. The LY-6 locus: a multigene family encoding phosphatidylinositol-anchored membrane proteins concerned with T-cell activation. Immunol Rev 1989;111:195-224.

4. Upadhyay G. Emerging Role of Lymphocyte Antigen-6 Family of Genes in Cancer and Immune Cells. Front Immunol 2019;10:819.

5. Lee PY, Wang JX, Parisini E, et al. Ly6 family proteins in neutrophil biology. J Leukoc Biol 2013;94:585-94.

6. Lee JW, Lee YS, Yoo KH, et al. LY-6K gene: a novel molecular marker for human breast cancer. Oncol Rep 2006;16:1211-4.

7. Suzuki H, Fukuhara M, Yamaura T, et al. Multiple therapeutic peptide vaccines consisting of combined novel cancer testis antigens and anti-angiogenic peptides for patients with non-small cell lung cancer. J Transl Med 2013;11:97.

8. AlHossiny M, Luo L, Frazier WR, et al. Ly6E/K Signaling to TGF $\beta$ Promotes Breast Cancer Progression, Immune Escape, and Drug Resistance. Cancer Res 2016;76:3376-86.

9. Choi SH, Kong HK, Park SY, et al. Metastatic effect of LY-6K gene in breast cancer cells. Int J Oncol 2009;35:601-7.

10. Luo L, McGarvey P, Madhavan S, et al. Distinct lymphocyte antigens 6 (Ly6) family members Ly6D, Ly6E, Ly6K and Ly6H drive tumorigenesis and clinical outcome. Oncotarget 2016;7:11165-93.

11. Matsuda R, Enokida H, Chiyomaru T, et al. LY6K is a novel molecular target in bladder cancer on basis of integrate genome-wide profiling. Br J Cancer 2011;104:376-86.

12. Ishikawa N, Takano A, Yasui $W$, et al. Cancer-testis antigen lymphocyte antigen 6 complex locus $\mathrm{K}$ is a serologic biomarker and a therapeutic target for lung and esophageal carcinomas. Cancer Res 2007;67:11601-11.

13. Liao XH, Xie Z, Guan CN. MiRNA-500a-3p inhibits cell proliferation and invasion by targeting lymphocyte antigen 6 complex locus K (LY6K) in human non-small cell lung cancer. Neoplasma 2018;65:673-82.

14. Galon J, Bruni D. Tumor Immunology and Tumor Evolution: Intertwined Histories. Immunity 2020;52:55-81.

15. Huarte E, Cubillos-Ruiz JR, Nesbeth YC, et al. Depletion of dendritic cells delays ovarian cancer progression by boosting antitumor immunity. Cancer Res 2008;68:7684-91.

16. Motz GT, Coukos G. Deciphering and reversing tumor immune suppression. Immunity 2013;39:61-73.

17. Chaplin DD. Overview of the immune response. J Allergy
Clin Immunol 2010;125:S3-23.

18. Jonsson AH, Yokoyama WM. Natural killer cell tolerance licensing and other mechanisms. Adv Immunol 2009; 101:27-79.

19. Son D, Kong HK, Kim Y, et al. Transgenic overexpression of human LY6K in mice suppresses mature T cell development in the thymus. Oncol Lett 2019;17:379-87.

20. Ishikawa H, Imano $M$, Shiraishi O, et al. Phase I clinical trial of vaccination with LY6K-derived peptide in patients with advanced gastric cancer. Gastric Cancer 2014;17:173-80.

21. Yoshitake Y, Fukuma D, Yuno A, et al. Phase II clinical trial of multiple peptide vaccination for advanced head and neck cancer patients revealed induction of immune responses and improved OS. Clin Cancer Res 2015;21:312-21.

22. Iwahashi M, Katsuda M, Nakamori M, et al. Vaccination with peptides derived from cancer-testis antigens in combination with CpG-7909 elicits strong specific CD8+ $T$ cell response in patients with metastatic esophageal squamous cell carcinoma. Cancer Sci 2010;101:2510-7.

23. de Nooij-van Dalen AG, van Dongen GA, Smeets SJ, et al. Characterization of the human Ly-6 antigens, the newly annotated member Ly-6K included, as molecular markers for head-and-neck squamous cell carcinoma. Int J Cancer 2003;103:768-74.

24. Maruyama M, Yoshitake H, Tsukamoto H, et al. Molecular expression of Ly6k, a putative glycosylphosphatidylinositol-anchored membrane protein on the mouse testicular germ cells. Biochem Biophys Res Commun 2010;402:75-81.

25. Letessier A, Sircoulomb F, Ginestier C, et al. Frequency, prognostic impact, and subtype association of 8p12, 8q24, $11 \mathrm{q} 13,12 \mathrm{p} 13,17 \mathrm{q} 12$, and 20q13 amplifications in breast cancers. BMC Cancer 2006;6:245.

26. Visapää H, Seligson D, Eeva M, et al. 8q24 amplification in transitional cell carcinoma of bladder. Appl Immunohistochem Mol Morphol 2003;11:33-6.

27. Fromont G, Godet J, Peyret A, et al. 8q24 amplification is associated with Myc expression and prostate cancer progression and is an independent predictor of recurrence after radical prostatectomy. Hum Pathol 2013;44:1617-23.

28. Loughner CL, Bruford EA, McAndrews MS, et al. Organization, evolution and functions of the human and mouse Ly6/uPAR family genes. Hum Genomics 2016;10:10.

29. Tomita Y, Yuno A, Tsukamoto H, et al. Identification of immunogenic LY6K long peptide encompassing both CD4+ and CD8+ T-cell epitopes and eliciting CD4+ 
T-cell immunity in patients with malignant disease. Oncoimmunology 2014;3:e28100.

30. Xu J, Liu H, Yang Y, et al. Genome-Wide Profiling of Cervical RNA-Binding Proteins Identifies Human Papillomavirus Regulation of RNASEH2A Expression by Viral E7 and E2F1. mBio 2019;10:02687-18.

31. Kong HK, Yoon S, Park JH. The regulatory mechanism of the LY6K gene expression in human breast cancer cells. J Biol Chem 2012;287:38889-900.

32. Sastry NG, Wan X, Huang T, et al. LY6K promotes glioblastoma tumorigenicity via CAV-1-mediated ERK1/2 signaling enhancement. Neuro Oncol 2020;22:1315-26.

33. Kong HK, Park SJ, Kim YS, et al. Epigenetic activation of LY6K predicts the presence of metastasis and poor prognosis in breast carcinoma. Oncotarget 2016;7:55677-89.

34. Kim YS, Park SJ, Lee YS, et al. miRNAs involved in LY6K and estrogen receptor $\alpha$ contribute to tamoxifen-susceptibility in breast cancer. Oncotarget 2016;7:42261-73.

35. Zhang B, Zhang Z, Zhang X, et al. Serological antibodies against LY6K as a diagnostic biomarker in esophageal squamous cell carcinoma. Biomarkers 2012;17:372-8.

36. Kono K, Mizukami Y, Daigo Y, et al. Vaccination with multiple peptides derived from novel cancer-testis antigens can induce specific T-cell responses and clinical responses in advanced esophageal cancer. Cancer Sci 2009;100:1502-9.

37. Ishikawa $H$, Imano $M$, Shiraishi $O$, et al. Phase I clinical trial of vaccination with URLC10-derived peptide for patients with advanced esophageal cancer. Esophagus 2012;9:105-12.

38. University S. Safety and Efficacy Study of Epitope Peptide To Treat HLA-A*02 Disease Controlled Advanced Nonsmall Cell Lung Cancer. 2016.

39. University S. Safety Study of Peptide Cancer Vaccine To Treat HLA-A*24-positive Advanced Non-small Cell Lung Cancer. 2013.

40. Sundar R, Rha SY, Yamaue H, et al. A phase I/Ib study of OTSGC-A24 combined peptide vaccine in advanced gastric cancer. BMC Cancer 2018;18:332.

41. Hospital AU. Gemcitabine With Peptide Vaccine Therapy in Treating Patients With Bile Duct Cancer. 2012.

42. University T. Histocompatibility Leukocyte Antigen (HLA)-A*0201 Restricted Peptide Vaccine Therapy in Patients With Esophageal Cancer. 2009.

43. Aruga A, Takeshita N, Kotera Y, et al. Long-term Vaccination with Multiple Peptides Derived from Cancer-
Testis Antigens Can Maintain a Specific T-cell Response and Achieve Disease Stability in Advanced Biliary Tract Cancer. Clin Cancer Res 2013;19:2224-31.

44. University T. Histocompatibility Leukocyte Antigen (HLA)-A*0201 Restricted Peptide Vaccine Therapy in Patients With Gastric Cancer. 2009.

45. University T. Histocompatibility Leukocyte Antigen (HLA)-A*2402 Restricted Peptide Vaccine Therapy in Patients With Non-Small Cell Lung Cancer. 2009.

46. Fujiwara Y, Okada K, Omori T, et al. Multiple therapeutic peptide vaccines for patients with advanced gastric cancer. Int J Oncol 2017;50:1655-62.

47. Higashihara Y, Kato J, Nagahara A, et al. Phase I clinical trial of peptide vaccination with URLC10 and VEGFR1 epitope peptides in patients with advanced gastric cancer. Int J Oncol 2014;44:662-8.

48. Iinuma H, Fukushima R, Inaba T, et al. Phase I clinical study of multiple epitope peptide vaccine combined with chemoradiation therapy in esophageal cancer patients. J Transl Med 2014;12:84.

49. Murahashi M, Hijikata Y, Yamada K, et al. Phase I clinical trial of a five-peptide cancer vaccine combined with cyclophosphamide in advanced solid tumors. Clin Immunol 2016;166-167:48-58.

50. Schroeder EB, Moore K, Manson SM, et al. An Interactive Voice Response and Text Message Intervention to Improve Blood Pressure Control Among Individuals With Hypertension Receiving Care at an Urban Indian Health Organization: Protocol and Baseline Characteristics of a Pragmatic Randomized Controlled Trial. JMIR Res Protoc 2019;8:e11794.

51. Schroeder EB, Moore KR, Manson SM, et al. A randomized clinical trial of an interactive voice response and text message intervention for individuals with hypertension. J Clin Hypertens (Greenwich) 2020;22:1228-38.

52. Takeuchi S, Kagabu M, Shoji T, et al. Anti-cancer immunotherapy using cancer-derived multiple epitopepeptides cocktail vaccination clinical studies in patients with refractory/persistent disease of uterine cervical cancer and ovarian cancer phase 2. Oncoimmunology 2020;9:1838189.

53. Kono K, Iinuma H, Akutsu Y, et al. Phase II cancer vaccination trial with multiple peptides derived from novel oncoantigens against advanced esophageal cancer. J Clin Oncol 2011;29:abstr 2504.

54. Kono K, Iinuma H, Akutsu Y, et al. Multicenter, phase II clinical trial of cancer vaccination for advanced esophageal 
cancer with three peptides derived from novel cancer-testis antigens. J Transl Med 2012;10:141.

55. Morse MA, Gwin WR 3rd, Mitchell DA. Vaccine Therapies for Cancer: Then and Now. Target Oncol 2021;16:121-52.

56. Antonelli AC, Binyamin A, Hohl TM, et al. Bacterial immunotherapy for cancer induces CD4-dependent tumor-specific immunity through tumor-intrinsic interferon- $\gamma$ signaling. Proc Natl Acad Sci U S A

Cite this article as: Guo D, Liu Y, Jiang Y, Zheng S, Xu T, Zhu J, Chen P, Huang P, Zhang Y. A narrative review of the emerging role of lymphocyte antigen 6 complex locus $\mathrm{K}$ in cancer: from basic research to clinical practice. Ann Transl Med 2022;10(1):26. doi: 10.21037/atm-21-5831
2020;117:18627-37.

57. Sahin U, Oehm P, Derhovanessian E, et al. An RNA vaccine drives immunity in checkpoint-inhibitor-treated melanoma. Nature 2020;585:107-12.

58. Schwartzentruber DJ, Lawson DH, Richards JM, et al. gp100 peptide vaccine and interleukin-2 in patients with advanced melanoma. N Engl J Med 2011;364:2119-27.

(English Language Editor: C. Betlazar-Maseh) 